\title{
Internet utility evaluation of a multi-start particle swarm optimization beamformer in a partial joint processing cellular network
}

\begin{abstract}
Joint processing (JP) is a powerful mechanism in alleviating inter-cell interference (ICI) and maximizing data rate of cell-edge users. However, JP puts tremendous load on both feedback and backhaul overhead, thus partial JP was proposed to overcome high overhead signaling. Unlike linear techniques such as zero-forcing (ZF), achieving an equivalent backhaul reduction and presenting a totally free ICI system based on limited feedback channel state information can be done by designing and implementing a multi-start particle swarm optimization algorithm (MSPSOA) as a beamforming (BF) mechanism. The performance of the proposed MSPSOA and ZF BF is evaluated based on the acquired spectral efficiency and utility of internet application such as hard real-time, adaptive and elastic applications. The simulation results shows that, the proposed MSPSOA BF achieves spectral efficiency up to $69.5 \%$ compared to $\mathrm{ZF} \mathrm{BF}$, and it maintains total utility for high data rate requirements of three common internet traffic such as hard real-time, adaptive and elastic applications.
\end{abstract}

Keyword: Beamforming; Interference avoidance; Joint processing; Particle swarm; Utility functions; Zero-forcing 